\title{
Alien hand, the Free Will and Arnold Pick
}

Alien hand phenomena (AHP) has had several meanings in neurology, but lately it has been most frequently used to describe the involuntary complex movements in corticobasal degeneration (CBD). C.M. Fisher, ${ }^{1}$ one of the masters of descriptive neurology, traces the history, definitions and interpretations of these phenomena in this issue. He also adds six of his own cases of corticobasal degeneration syndrome, which is a new term for the extrapyramidal presentation of Pick's disease. He goes further, however, by introducing philosophical and physiological issues about free will and movements.

The initial term used in callosal disconnection patients, whose nondominant hand interfered with the voluntary movements of the dominant hand was "diagonistic dyspraxia". Brion and Jedynak $^{3}$ subsequently coined the term of the sign of the "alien hand" for their cases. Many subsequent descriptions adopted the term, including the case reports of CBD. Fisher summarizes this literature, grouping the varieties according to etiology, such as callosal sections, tumors, anterior cerebral artery infarct and aneurysm, etc. Some of these lesions appear to involve the frontal lobe, and some of the symptomatology such as groping, compulsive manipulation of objects, and grasp reflex suggest frontal lobe mechanisms, especially the damage to the supplementary motor area as the cause.

Although Brion and Jedynak's cases misidentified their hand when it was palpated behind their back, the denial of ownership of the hand is much more common in severe cases of neglect and anosognosia of the nondominant hand. This sensorimotor neglect form of AHP could be added as yet another variety. In fact, there is some evidence that $\mathrm{CBD}$ has a major parietal component to it and in some cases sensory deficits conceivably contribute to this phenomena.

Although the first two cases described under corticodentatonigral degeneration ${ }^{4}$ had AHP, this is by no means consistently described in CBD. Some patients only show levitation, odd posturing and grasp reflex. Uncontrollable wandering of the hand, crossing the midline and interfering with movements of the contralateral limb and grasping objects is not always present. Nevertheless, AHP seems to be common in CBD, even though precise descriptions or criteria for identification of the phenomena are not always provided.

Fisher's 5/6 cases were remarkable because they had bilateral AHP phenomena. These patients had complex, unwilled motor acts including intermanual conflict, mirror movements, interference, enabling synkinesis, reversal of complex acts and pushing aside one limb by the other, instead of cooperating. Relatively simple unwilled, unskilled quasi reflex actions including reaching out, groping, grasping, inability to release, withdrawal of the limb, compulsive utilization of tools, and other utilization behaviour were also a feature.

Bimanual movements are often integrating and cooperative in nature and failure to coordinate these movements is interpreted as disconnection. In intermanual conflict each hand seems to carry out on its own. Often it is the nondominant limb that shows unintended, unwilled performance. The evidence, as so clearly summarized by Fisher points to the lesions of the supplementary area and interruption of the corpus callosum.

The philosophical issue of the role of "the will" in movements is an important issue in describing the AHP. Patients often provide verbal evidence that the interfering hand is acting in an unwilled fashion "on its own." Hence the term, alien hand. Various physiological evidence indicates that normal movements are preceded by electrical activity even before the conscious decision is made to act. According to the reflex theory of movement, all movements are reflexes of one sort or another to some sensory stimulus and as the act unfolds it is recorded as an ideation or a thought which provides the impression that the act was willed. It seems, however, that there are motor phenomena that are not part of the "will". These unwilled movements can be complex and well-executed and they occur while the patient is aware of the movement but still considers it unwilled. The readiness potentials recorded, preceding movements, may reflect a physiological contribution to will. Fisher postulates that the lesion of the supplementary motor area (SMA) prevents this basic component and he supports this by evidence from the literature. ${ }^{5}$ Interestingly and somewhat arguably, he comes out at the end against the concept of "free will."

Although the pathological description is not a major part of Fisher's article, 4/6 of his cases were pathologically confirmed. He briefly touches upon the increasing evidence that CBD is part of the Pick complex and that Pick's disease is often the underlying pathology with the clinical syndrome of CBD. ${ }^{6}$

\section{A. Kertesz}

\section{REFERENCES}

1. Fisher CM. Alien hand phenomena: a review with the addition of five personal cases. Can J Neurol Sci 2000;27:192-203.

2. Akelaitis AJ. Studies on the corpus callosum IV diagonistic dyspraxia in epileptics following partial and complete section of the corpus callosum. Am J Psychiatry 1944;101:594-599.

3. Brion S, Jedynak CP. Troubles du transfert interhémisphérique (callosal disconnection) a propos de 3 observations de tumeurs du corps calleux. Le signe de la main étrangère. Rev Neurol 1972; 126:308-359.

4. Rebeiz JJ, Kolodny EH, Richardson EP, Jr. Corticodentatonigral degeneration with neuronal achromasia. Arch Neurol 1968;18: 20-33.

5. Tanaka Y, Iwasa H, Yoshida M. Diagnostic dyspraxia: case report and movement related potentials. Neurology 1990;40:657-661.

6. Kertesz A, Munoz DG. Pick's Disease and Pick Complex. New York: Wiley-Liss, Inc. 1998. 